\title{
Tumores del estroma gastrointestinal
}

\section{Cambio conceptual de los tumores del estroma gastrointestinal (GISTs) y criterios de malignidad}

\author{
L. Ortega Medina, M. Saiz-Pardo Sanz
}

\section{Introducción}

Los tumores del estroma gastrointestinal (GIST), aunque son sólo el $2 \%$ de las neoplasias del tubo digestivo, son las neoplasias mesenquimales más frecuentes del tracto gastrointestinal $^{1,2}$. El término GIST (3) es de reciente introducción y se utiliza para denominar a un grupo de neoplasias que hasta ahora y por su aspecto morfológico se consideraban de origen muscular y ocasionalmente neural. Este cambio conceptual ha surgido porque aunque su morfología es similar a la de los tumores musculares, responden peor al tratamiento que los leiomiosarcomas de otras localizaciones. De hecho, su respuesta a la quimioterapia y a la radioterapia es prácticamente nula, y además porque con frecuencia no expresan marcadores musculares e incluso pueden mostrar marcadores neurales. Su perfil inmunohistoquímico es similar al de las células intersticiales de Cajal, con positividad para CD1 17 (c-kit: receptor de tirosín-quinasa), CD34 y vimentina 3,4 lo que sugiere un origen en dichas células ${ }^{5}$.

\section{Evolución histórica y concepto}

Desde las primeras descripciones de Golden y Stout ${ }^{6}$, este grupo de lesiones mesenquimales se consideraron de origen muscular y recibieron nombres como leiomioma, leiomioma celular, leiomioma epitelioide, leiomioblastoma, leiomioma bizarro y leiomiosarcoma. No es, sin embargo, hasta la utilización de la inmunohistoquímica y la subsiguiente demostración de la ausencia de marcadores musculares y la presencia ocasional de marcadores neurales, que surge de la mano de Mazur y Clark ${ }^{3}$ el término de tumores del estroma. Poco después se introdujo el término de plexosarcoma ${ }^{7}$, para lesiones que mostraban diferenciación neuronal y que pasaría a llamarse tumor nervioso autonómico gastrointestinal. Poste-

Servicio de Anatomía Patológica

Hospital Clínico San Carlos

Madrid riormente, se comprobó que estas neoplasias expresaban CD34 ${ }^{8}$, si bien este dato era de poca utilidad para establecer criterios diagnósticos y/o histogenéticos dada la inespecificidad de este marcador. No fue hasta la demostración de que estas neoplasias contenían mutaciones del protoncogén KIT, que se asocia con la expresión de proteína ${ }^{4,9}$, que se definieron estos tumores como una entidad con características clinicopatológicas propias. De tal forma que actualmente se denominan GISTs a los tumores mesenquimales CD117 positivos, fusiformes o epitelioides, primarios del tracto gastrointestinal, epiplon, mesenterio y retroperitoneo ${ }^{10}$.

\section{Anatomía patológica}

Los GISTs aparecen en la pared del tubo digestivo: estómago (50-60\%), intestino delgado (20-30\%), intestino grueso $(10 \%)$ y esófago $(5 \%)$ y ocasionalmente en epiplon, mesenterio y retroperitoneo $(5 \%)^{2,9}$.

Su aspecto macroscópico depende del tamaño, que oscila entre los 0.8 y los $38 \mathrm{~cm}^{10,11}$. Los GISTs pequeños aparecen como nódulos serosos, intramurales o submucosos; los grandes suelen tener crecimiento exofítico hacia la luz y ulceran la mucosa hasta en el $20-30 \%$ de los casos, aunque esto no implica agresividad. También pueden protruir hacia la superficie serosa, con gran componente extraparietal que enmascara su origen digestivo. Al corte están bien delimitados, son lobulados y de aspecto arremolinado. En algunos casos, especialmente en las lesiones malignas, aunque sin que estos hallazgos puedan considerarse como diagnósticos de malignidad, pueden encontrarse áreas de necrosis, hemorragia y de degeneración quística (Fig. 1).

El aspecto microscópico es variable y es consecuencia de los distintos tipos celulares que pueden aparecer en los GISTs, de forma que se pueden distinguir tres categorías: de células fusiformes $(77 \%)$, de células epitelioides $(8 \%)$ y mixtos $(15 \%)^{10}$. Los GISTs de células fusiformes (Fig. 2) están constituidos por células alargadas de citoplasma pálido y eosinófilo y, a menudo, aspecto fibrilar y sincitial, que se disponen en haces entrecruzados con patrón de crecimiento estoriforme y formación de empalizadas nucleares. Los núcleos son ovoideos y con frecuentes vacuolas citoplásmicas paranucleares, lo que fue interpretado en el pasado, junto con la formación de empalizadas, como una prueba de su diferenciación muscular. Los GISTs epitelioides (Fig. 3) están constitui- 


\section{Ortega Medina y M. Saiz-Pardo Sanz}

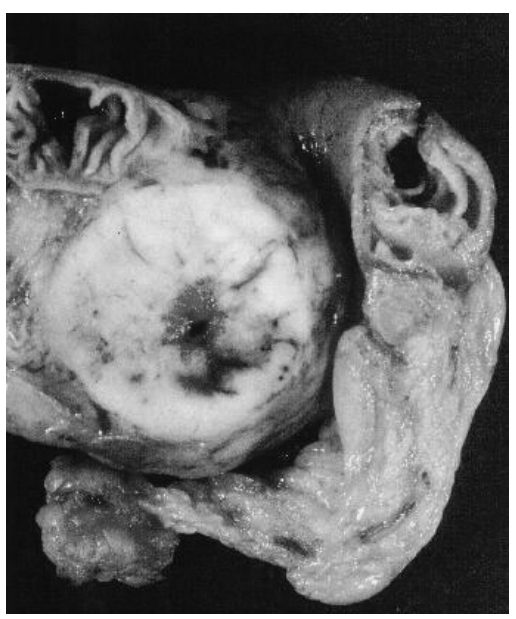

Fig. 1. GIST maligno de intestino delgado. Nódulo de aspecto arremolinado con focos de hemorragia.

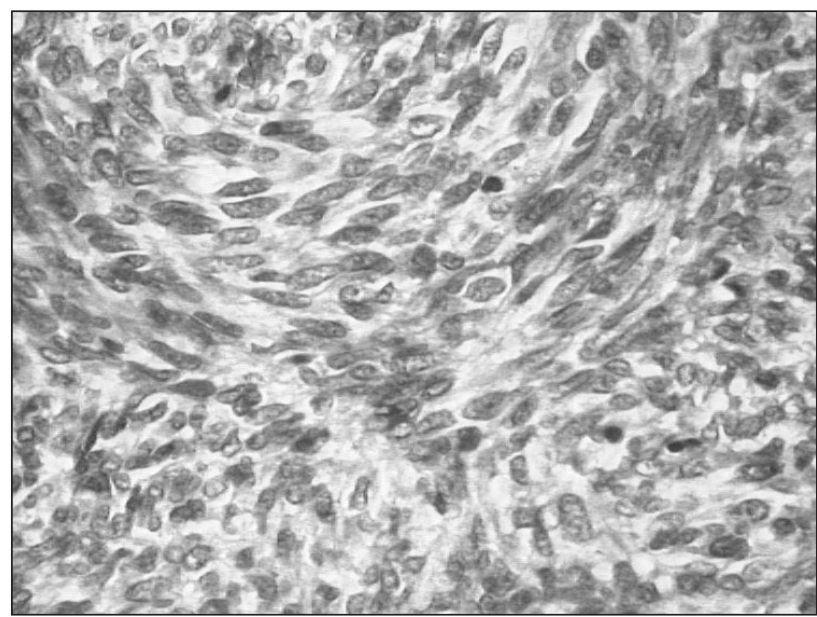

Fig. 2. GIST fusiforme. Células alargadas dispuestas en fascículos (HE, 200x).

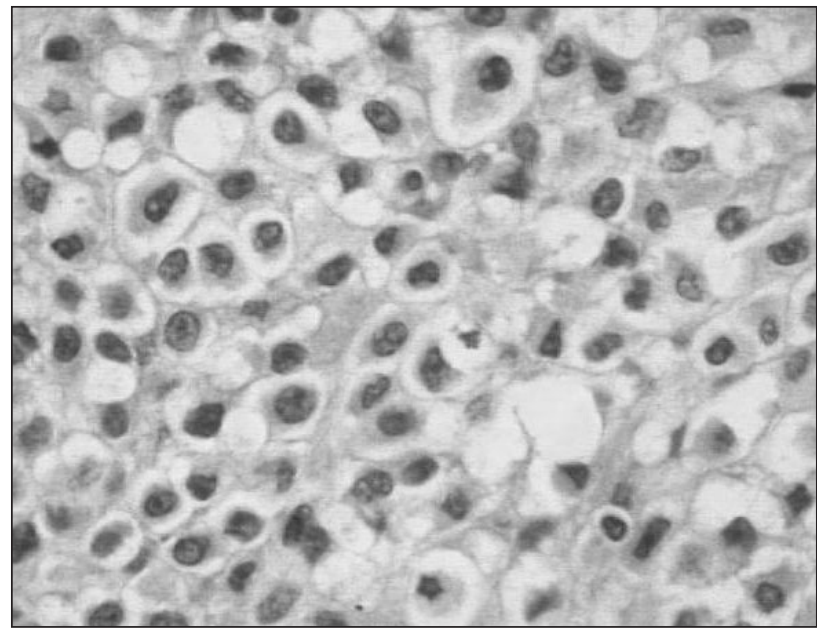

Fig. 3. GIST epitelioide. Células poligonales de citoplasma vacuolado (HE 200x). dos por células de citoplasma eosinófilo o claro, a veces vacuolado, recordando a las células en anillo de sello de los carcinomas. Además, tienden a crecer formando pequeños nidos, lo que acentúa aún más su aspecto epitelial. Los GISTs mixtos están constituidos por una mezcla de células epitelioides y fusiformes, con una transición brusca entre ambos tipos celulares o con un tercer tipo ovoideo, intermedio entre ambos $^{12}$.

Otros rasgos estructurales son la presencia de estroma hialino, rico en fibras colágenas, el infiltrado inflamatorio linfocitario o la existencia de estroma mixoide, con cambios microquísticos que recuerdan, junto con las empalizadas, a los schwannomas. Por último, un rasgo característico y más frecuente en los GISTs de intestino delgado, donde aparecen hasta en el $55.5 \%$ de los $\operatorname{casos}^{10}$, son las fibras en madeja ("skeinoid fibers"), que son estructuras fibrilares PAS positivas e intensamente eosinófilas, que están compuestas por fibras colágenas y que aunque fueron interpretadas como un signo de diferenciación neural, parece que no tienen ningún significado histogenético ${ }^{5,12}$.

Con microscopio electrónico se detectan células con diferenciación muscular lisa (vesículas de pinocitosis, cuerpos densos subplasmalémicos y abundantes microfilamentos), diferenciación neural (uniones intercelulares primitivas, numerosos microtúbulos y gránulos de tipo neurosecretor), ambos tipos de diferenciación o ninguno de ellos ${ }^{13}$. Estas características ultraestructurales, junto al inmunofenotipo fue la causa de que se propusiera una clasificación de los GISTs dependiendo de su diferenciación, en cuatro grupos: GISTs con diferenciación muscular lisa, GISTs con diferenciación neural, GISTs con diferenciación dual muscular y neural y GISTs sin diferenciación. Dicha clasificación ha sido abandonada en la actualidad, porque consideraba a todas las neoplasias con diferenciación neural, dual o sin diferenciación, como malignas o potencialmente malignas y porque no contemplaba la histogénesis de los GISTs.

Los GISTs pueden expresar gran cantidad de antígenos; el más constante es el CD1 17 o c-kit que se encuentra en prácticamente el $100 \%$ de los casos, con un patrón de tinción difuso o en el aparato de Golgi (Fig. 4). También se observa

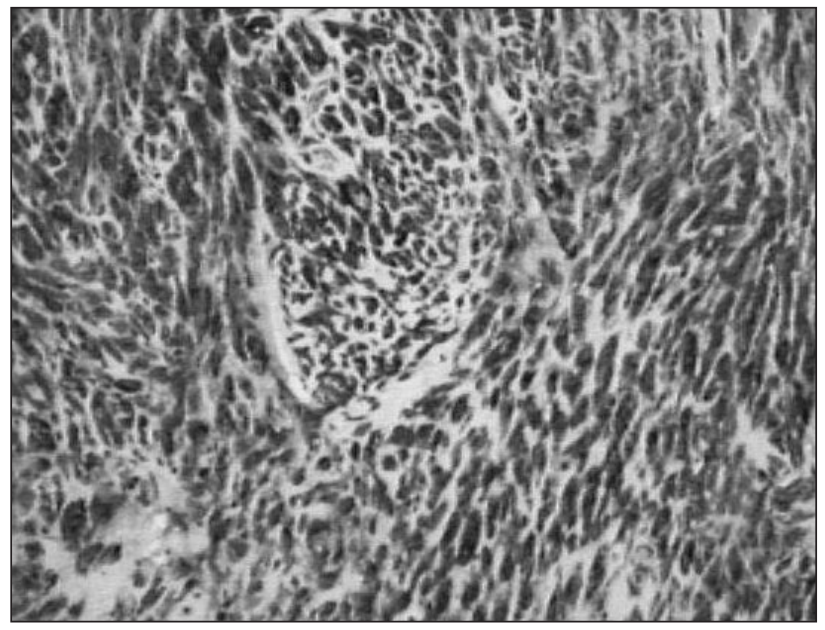

Fig. 4. Intensa positividad difusa para c-kit (CDI 17) en un GIST maligno (PAP, c-kit 200x). 
Oncología, 2004; 27 (4):237-241

positividad para CD34 (70-90\%), actina (20-30\%), proteína S-100 (8-10\%) y desmina (2-4\%) $)^{1,10}$. De forma esporádica también se ha descrito positividad para enolasa neuronal específica, neurofilamentos, cromogranina e incluso citoqueratina ${ }^{13}$.

\section{Diagnóstico diferencial}

El diagnóstico diferencial se plantea con neoplasias similares morfológicamente o que son positivas para CD34 y por otro lado con tumores que pueden expresar c-kit. Entre las primeras tenemos a las neoplasias fibrohistiocitarias, a las de vaina nerviosa periférica (schwannoma y tumor maligno de vaina nerviosa periférica), a las musculares lisas (leiomioma que es más frecuente en esófago y en relación a la muscularis mucosae de colon y recto y leiomiosarcoma, más frecuentes en retroperitoneo y raros en tubo digestivo), al tumor fibroso solitario de membrana serosa, al sarcoma de Kaposi (6), a los adenocarcinomas poco diferenciados y a lesiones proliferativas como el pólipo fibroide inflamatorio y los tumores inflamatorios miofibroblásticos. En el segundo grupo de entidades tenemos a los liposarcomas y a los melanomas metastásicos, que por su aspecto variable pueden semejar, además, un GIST ${ }^{14}$. El problema fundamental del diagnóstico se produce ante aquellas lesiones morfológicamente típicas de GIST que tienen negatividad para c-kit. Esta puede deberse a problema de muestreo en neoplasias con positividad focal, especialmente si la biopsia es endoscópica, a que el tejido sea inmunohistoguímicamente inerte por procesamiento inadecuado o tras almacenamiento prolongado, a pérdida de expresión antigénica, como puede ocurrir tras tratamiento o, por último, porque haya un porcentaje pequeño $(<2 \%)$ de tumores que, aún con características morfológicas típicas, carezca de mutaciones y/o de sobreexpresión de kit ${ }^{12}$. Algunos de estos casos tienen mutaciones del receptor (del factor de crecimiento derivado de las plaquetas) ${ }^{15}$.

\section{Criterios de malignidad}

Uno de los retos en el estudio de los GISTs es establecer la agresividad y comportamiento biológico. Como es evidente, el criterio de malignidad más fiable es la presencia de metastásis y/o invasión de los órganos adyacentes, aunque habitualmente, en el momento del diagnóstico, la diseminación todavía no se ha producido, por lo que el patólogo debe establecer qué características son útiles para pronosticar la evolución de la neoplasia. Ciertos rasgos que en otras neoplasias son indicativos de agresividad, son de importancia dudosa en los GISTs. Así, no está clara la importancia de la densidad celular ni de la atipia. Tampoco tiene relación con el comportamiento la presencia de ulceración mucosa, ni la infiltración de la capa muscular, que puede darse por el origen de los GISTs en las células intersticiales de Cajal y no por una auténtica capacidad infiltrativa de la neoplasia ${ }^{16}$. Sin embargo, la invasión mucosa parece asociarse con las lesiones malignas ${ }^{17}$. Se observa como un crecimiento difuso, intersticial, entre las glándulas. No se debe considerar como invasión mucosa la mera presencia de ulceración. Si se sigue este criterio, la invasión mucosa es diagnóstica de malignidad, por lo que puede ser de utilidad en el caso de biopsias
TABLA I

Criterios de Amin et al. ${ }^{18}$ de malignidad en GISTs

Benignos: Menos de 5 mitosis / 50 CGA y tamaño $<5 \mathrm{~cm}$ Borderline: Mismo número de mitosis. Tamaño mayor de $5 \mathrm{~cm}$ Malignos: Más de 5 mitosis /50 CGA y cualquier tamaño

TABLA II

Criterios de Miettinen et al. ${ }^{18}$ de malignidad en GISTs

Probablemente benignos:

- Tumores intestinales: Diámetro máximo $<2 \mathrm{~cm}$ y no más de 5 mitosis/50 CGA

- Tumores gástricos: Diámetro máximo $<5 \mathrm{~cm}$ y no más de 5 mitosis/50 CGA

Probablemente malignos:

- Tumores intestinales: Diámetro máximo $>5 \mathrm{~cm}$ o más de $5 \mathrm{mi}-$ tosis/50 CGA

- Tumores gástricos: Diámetro máximo $>10 \mathrm{~cm}$ o más de 5 mitosis/50 CGA

Bajo potencial maligno o potencial incierto:

- Tumores intestinales: Diámetro máximo $>2 \mathrm{~cm}$ pero $<5 \mathrm{~cm}$ y no más de 5 mitosis/50 CGA

- Tumores gástricos: Diámetro máximo $>5 \mathrm{~cm}$ pero $<10 \mathrm{~cm}$ y no más de 5 mitosis/50 CGA

endoscópicas, si bien su rareza limita su valor práctico ${ }^{16}$. Otro dato que ha sido barajado es la localización, ya que la incidencia de lesiones agresivas varía, de forma que los GISTs de estómago son más frecuentemente benignos que malignos, tendencia que se invierte en el intestino ${ }^{16}$. En cualquier caso parece que los GISTs se pueden estratificar en lesiones de muy bajo, bajo, intermedio y alto riesgo atendiendo a su tamaño, definiendo éste como su diámetro máximo y al número de mitosis. El primer paso en este sentido fue dado por Amin et al. ${ }^{18}$, estableciendo como límites clave en la clasificación de estos tumores los $5 \mathrm{~cm}$ de tamaño y las $5 \mathrm{mi}$ tosis por 50 campos de gran aumento (Tabla I). Pero estos criterios no tenían en cuenta la localización de las lesiones, por lo que Miettinen et al. ${ }^{16}$ introducen la localización como un tercer parámetro en la evaluación de la malignidad (Tabla II), aunque otros autores ${ }^{19}$ no consideran que la localización sea importante para establecer un pronóstico. En la actualidad, y tras una Conferencia de Consenso, otros autores consideran que no es conveniente utilizar el término de benigno para ningún GIST, asumiendo que todos ellos pueden llegar a tener comportamiento agresivo'2, por lo que es más adecuado hablar de riesgo de conducta agresiva, si bien los criterios para establecer dicho riesgo siguen siendo el tamaño del tumor y el índice mitótico (Tabla III). Estos autores consideran, además, que en el caso de los GISTs intestinales el criterio de tamaño debe ser $1 \circ 2 \mathrm{~cm}$ menor. La ventaja de estos criterios es que son fácilmente aplicables en cualquier laboratorio de Anatomía Patológica.

Otros estudios han intentado establecer criterios inmunohistoquímicos de agresividad. Así, por ejemplo, no hay 
TABLA III

\section{Criterios de Fletcher et al. ${ }^{12}$ de riesgo de conducta agresiva en GISTs}

\begin{tabular}{lll}
\hline & Tamaño & Número de mitosis \\
\hline Muy bajo riesgo & $<2 \mathrm{~cm}$ & $<5 / 50$ CGA \\
Bajo riesgo & $2-5 \mathrm{~cm}$ & $<5 / 50 \mathrm{CGA}$ \\
Riesgo intermedio & $<5 \mathrm{~cm}$ & $6-10 / 50 \mathrm{CGA}$ \\
& $5-10 \mathrm{~cm}$ & $<5 / 50 \mathrm{CGA}$ \\
Alto riesgo & $>5 \mathrm{~cm}$ & $5 / 50$ CGA \\
& $10 \mathrm{~cm}$ & Cualquier índice mitótico \\
& Cualquier tamaño & $>10 / 50$ CGA \\
\hline
\end{tabular}

correlación entre la inmunotinción para CD34 y el comportamiento $^{16}$. La positividad para p53, Bcl-2 y ciclina D1 no es útil para establecer el pronóstico ${ }^{20}$; sin embargo, la expresión del factor de crecimiento endotelial vascular (VEGF), indicativa de una estimulación de la angiogénesis y las alteraciones de la p16 se asocian a un peor pronóstico ${ }^{21,22}$.

Otra de las técnicas más utilizadas ha sido la valoración del índice de proliferación nuclear evidenciado por la tinción para MIB-1 (Ki67), de forma que un índice del 10\% o más, se asocia a peor pronóstico ${ }^{23}$.

También se han estudiado los marcadores genéticos como factores pronósticos. Se han usado tres tipos de análisis. En primer lugar el estudio de pérdidas y ganancias de material genético mediante hibridación genómica comparativa y estudio de pérdida de heterocigosidad. De esta forma, se ha visto que los GISTs benignos tienen un número significativamente inferior de copias de ADN que los malignos ${ }^{24}$. Por otra parte, las delecciones de $9 p$ y $9 q$ y las pérdidas alélicas de $22 q^{25}$ son más frecuentes en las lesiones malignas, que además tienen un número medio de aberraciones cromosómicas más alto. Hay sobreexpresión de ciertos genes en los GISTs malignos como son VIL2, COL8A1, CCNB2, HMG2, TSG101, CENp-F, FAK y DYRK2 ${ }^{26}$. En esta misma línea se ha visto que la metilación de E-cadherina se asocia a peor supervivencia a los 5 años ${ }^{27}$. También se ha valorado la actividad de telomerasa, de forma que sólo los GISTs malignos mostraron actividad de telomerasa, si bien no todos los malignos demostraron dicha actividad ${ }^{28}$.

En último lugar se ha investigado la relación entre las mutaciones de c-kit y la agresividad, aunque el valor pronóstico de dichas mutaciones no está claro, y aunque se han relacionado las mutaciones de los exones 11 y 9 con tumores de mayor tamaño, con índices de recurrencia mayor y menor supervivencia a los 5 años, hay datos discordantes en la literatura, por lo que se requieren nuevas investigaciones para dilucidar la importancia pronóstica de dichas mutaciones ${ }^{16}$. Recientemente el grupo de Miettinen ${ }^{29}$ ha encontrado que cierto tipo de mutaciones del dominio yuxtamembrana son más frecuentes en estómago y tienen mejor pronóstico.

\section{Conclusiones}

Los estudios inmunohistoquímicos y de patología molecular han revolucionado el concepto y diagnóstico de los
GISTs. Esto ha permitido una mejor clasificación histogenética de las neoplasias mesenquimales digestivas y la aplicación de un tratamiento específico en este grupo de tumores. El patólogo es el responsable del diagnóstico de estas neoplasias, que se debe basar en un estudio morfológico convencional $y$ un estudio inmunohistoquímico con demostración de c-kit y debe también establecer el riesgo de conducta agresiva de las lesiones, atendiendo al tamaño del tumor y al índice mitótico.

\author{
Correspondencia: \\ Dr. L. Ortega Medina \\ Servicio de Anatomía Patológica \\ Hospital Clínico San Carlos \\ $\mathrm{C} /$ Martín Lagos, s/n \\ E-28040 Madrid \\ E-mail: lortega.hcsc@salud.madrid.org
}

\section{Bibliografía}

1. Thomas RM, Sobin LH. Gastrointestinal cancer: incidence and prognosis by histologic type. SEER population-based data: 1978-1987. Cancer 1995; 75:154-70.

2. Miettinen $M$, Majidi M, Lasota J. Pathology and diagnostic criteria of gastrointestinal stromal tumors (GISTs): a review. Eur J Cancer 2002; 38 Suppl 5:S39-51.

3. Mazur MT, Clark HB. Gastric stromal tumors. Reappraisal of histogenesis. Am J Surg Pathol 1983; 7:507-19.

4. Kindblom LG, Remotti HE, Aldenborg F, Meis-Kindblom JM. Gastrointestinal pacemaker cell tumor (GIPACT): gastrointestinal stromal tumors show phenotypic characteristics of the interstitial cells of Cajal. Am J Pathol 1998; 152:1259-69.

5. Sircar K, Hewlett BR, Huizinga JD, Chorneyko K, Berezin I, Riddell RH. Interstitial cells of Cajal as precursors of gastrointestinal stromal tumors. Am J Surg Pathol 1999; 23:377-89.

6. Golden T, Stout AP. Smooth muscle tumours of the gastrointestinal tract and retroperitoneal tissues. Surg Gynecol Obstet. $1941 ; 73: 704-10$.

7. Herrera GA, Pinto de Moraes H, Grizzle WE, Han SG. Malignant small bowel neoplasm of enteric plexus derivation (plexosarcoma). Light and electron microscopic study confirming the origin of the neoplasm. Dig Dis Sci 1984; 29:275-84.

8. Miettinen M, Virolainen M, Sarlomo-Rikala M. Gastrointestinal stromal tumors: value of CD34 antigen in their identification and separation from true leiomyomas and schwannomas. Am J Surg Pathol 1995; 19:207-16.

9. Hirota $S$, Isozaki K, Moriyama $Y$, Hashimoto K, Nishida T, Ishiguro S, Kawano K, Hanada M, Kurata A, Takeda M, Muhammad Tunio G, Matsuzawa Y, Kanakura Y, Shinomura Y, Kitamura $Y$. Gain of function mutations of c-kit in human gastrointestinal stromal tumors. Science 1998; 279:577-80.

10. Miettinen $M$, Lasota J. Gastrointestinal stromal tumors-definition, clinical, histological, immunohistochemical, and molecular genetic features and differential diagnosis. Virchows Arch 2001; 438:1-12.

11. Kim CJ, Day S, Yeh KA. Gastrointestinal stromal tumors: analysis of clinical and pathologic factors. Am Surg 2001; 67:1357. 
12. Fletcher $C D$, Berman JJ, Corless $C$, Gorstein F, Lasota J, Longley BJ, Miettinen M, O'Leary TJ, Remotti H, Rubin BP, Shmookler B, Sobin LH, Weiss SW. Diagnosis of gastrointestinal stromal tumors: A consensus approach. Hum Pathol 2002; 33:459-65.

13. Erlandson RA, Klimstra DS, Woodruff JM. Subclassification of gastrointestinal stromal tumors based on evaluation by electron microscopy and immunohistochemistry. Ultrastruct Pathol 1996; 20:373-93.

14. Miettinen M, Sobin LH, Sarlomo-Rikala M. Immunohistochemical spectrum of GISTs at different sites and their differential diagnosis with a reference to CD1 17 (KIT). Mod Pathol 2000; 13:1134-42.

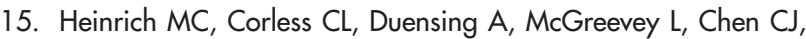
Joseph N, Singer S, Griffith DJ, Haley A, Town A, Demetri GD, Fletcher CDM, Fletcher JA. PDGFRA activating mutations in gastrointestinal stromal tumors. Science 2003; 299:708-10.

16. Miettinen M, El-Rifai W, H L Sobin L, Lasota J. Evaluation of malignancy and prognosis of gastrointestinal stromal tumors: a review. Hum Pathol 2002; 33:478-83.

17. Trupiano JK, Stewart RE, Misick C, Appelman HD, Goldblum JR. Gastric stromal tumors: a clinicopathologic study of $77 \mathrm{ca}-$ ses with correlation of features with nonaggressive and aggressive clinical behaviors. Am J Surg Pathol 2002; 26:705-14.

18. Amin MB, Ma CK, Linden MD, Kubus JJ, Zarbo RJ. Prognostic value of proliferating cell nuclear antigen index in gastric stromal tumors. Correlation with mitotic count and clinical outcome. Am J Clin Pathol 1993; 100:428-32.

19. Yan H, Marchettini P, Acherman YI, Gething SA, Brun E, Sugarbaker $\mathrm{PH}$. Prognostic assessment of gastrointestinal stromal tumor. Am J Clin Oncol 2003; 26:221-8.

20. Wong NA, Young R, Malcomson RD, Nayar AG, Jamieson LA, Save VE, Carey FA, Brewster DH, Han C, Al-Nafussi A. Prognostic indicators for gastrointestinal stromal tumours: a clinicopathological and immunohistochemical study of 108 resected cases of the stomach. Histopathology 2003; 43:118-26.

21. Takahashi R, Tanaka S, Kitadai Y, Sumii M, Yoshihara M, Haruma K, Chayama K. Expression of vascular endothelial growth factor and angiogenesis in gastrointestinal stromal tumor of the stomach. Oncology 2003; 64:266-74.
22. Schneider-Stock R, Boltze $C$, Lasota J, Miettinen M, Peters $B$, Pross $M$, Roessner A, Gunther T. High prognostic value of pl6INK4 alterations in gastrointestinal stromal tumors. J Clin Oncol 2003; 21:1688-97.

23. Toquet C, Le Neel JC, Guillou L, Renaudin K, Hamy A, Heymann MF, Simon-Valla S, Le Borgne J, Maugard C, Fiche M. Elevated (>or $=10 \%$ ) MIB-1 proliferative index correlates with poor outcome in gastric stromal tumor patients: a study of 35 cases. Dig Dis Sci 2002; 47:2247-53.

24. El-Rifai W, Sarlomo-Rikala M, Andersson LC, Knuutila S, Miettinen M.DNA sequence copy number changes in gastrointestinal stromal tumors: tumor progression and prognostic significance. Cancer Res 2000; 60:3899-903.

25. Kim NG, Kim JJ, Ahn JY, Seong CM, Noh SH, Kim CB, Min JS, $\operatorname{Kim~H}$. Putative chromosomal deletions on 9P, 9Q and 22Q occur preferentially in malignant gastrointestinal stromal tumors. Int J Cancer 2000; 85:633-8.

26. Koon N, Schneider-Stock R, Sarlomo-Rikala M, Lasota J, Smolkin M, Petroni G, Zaika A, Boltze C, Meyer F, Andersson L, Knuutila S, Miettinen M, El-Rifai W. Molecular targets for tumour progression in gastrointestinal stromal tumours. Gut 2004; 53:235-40.

27. House MG, Guo M, Efron DT, Lillemoe KD, Cameron JL, Syphard JE, Hooker CM, Abraham SC, Montgomery EA, Herman JG, Brock MV. Tumor suppressor gene hypermethylation as a predictor of gastric stromal tumor behavior. J Gastrointest Surg 2003; 7:1004-14.

28. Gunther T, Schneider-Stock R, Hackel C, Pross M, Schulz HU, Lippert $H$, Roessner A.Telomerase activity and expression of hTRT and hTR in gastrointestinal stromal tumors in comparison with extragastrointestinal sarcomas. Clin Cancer Res 2000; 6: 1811-8.

29. Lasota J, Dansonka-Mieszkowska A, Stachura T, SchneiderStock R, Kallajoki M, Steigen SE, Sarlomo-Rikala M, Boltze C, Kordek R, Roessner A, Stachura J, Miettinen M. Gastrointestinal stromal tumors with internal tandem duplications in $3^{\prime}$ end of KIT juxtamembrane domain occur predominantly in stomach and generally seem to have a favorable course. Mod Pathol 2003; 16:1257-64. 